\title{
TERAPIA COGNITIVO-COMPORTAMENTAL NO TRATAMENTO DA DEPENDÊNCIA QUÍMICA
}

\section{Cognitive-Behavioral Therapy in the treatment of Chemical Dependency}

\author{
Gesse Ávila ${ }^{1}$ \\ Maria Tereza Soratto²
}

\begin{abstract}
RESUMO
Uma das premissas fundamentais da Terapia cognitivo-comportamental é tornar o paciente seu próprio terapeuta. Pesquisa com objetivo de analisar publicações científicas sobre a eficácia da terapia cognitivo comportamental no tratamento da dependência química. Trata-se de uma pesquisa de revisão narrativa, de caráter descritivo, qualitativo, desenvolvido a partir de artigos indexados na Scielo (Scientific Eletronic Libraly Online); Bireme; BVS psi - Biblioteca Virtual em Saúde - Psicologia Brasil e Google acadêmico a fim de se identificar publicações recentes envolvendo a temática sobre a eficácia da terapia cognitivo comportamental no tratamento da dependência química. A Terapia cognitivo-comportamental é considerada uma ferramenta poderosa no tratamento da dependência química, dando ênfase às técnicas como a psicoeducação, sendo uma terapia eficaz na prevenção de recaídas.
\end{abstract}

Palavras-chave: Dependência Química; Substância Química; Terapia Cognitivo Comportamental; Psicologia

\begin{abstract}
One of the fundamental premises of cognitive-behavioral therapy is to make the patient your own therapist. Research aiming to analyze scientific publications on the

1 Psicólogo. Especialista em Terapia Cognitivo Comportamental (TCC) e Aplicabilidade nas Psicopatologias. UNESC - Universidade do Extremo Sul de Santa Catarina - Criciúma - SC - Brasil. Email: gesseavila@hotmail.com

${ }^{2}$ Enfermeira. Mestre em Educação. Professora do Curso de Enfermagem - UNESC - Universidade do Extremo Sul de Santa Catarina - Criciúma - SC - Brasil. Email: guiga@unesc.net
\end{abstract}

Revista Inova Saúde, Criciúma, vol. 10, n. 1, fev. 2020. 
effectiveness of cognitive behavioral therapy in the treatment of chemical dependency. This is a survey of review narrative, descriptive, qualitative character, developed from articles indexed in Scielo (Scientific Electronic Online Libraly); Bireme and Virtual Health Library-Psychology Brazil and Google Scholar to identify recent publications involving the theme about the effectiveness of cognitive behavioral therapy in the treatment of chemical dependency. Cognitive-behavioral therapy is considered to be a powerful tool in the treatment of chemical dependency, giving emphasis to techniques such as psychoeducation, being an effective therapy in relapse prevention.

Keywords: Chemical Dependence; Chemical substance; Cognitive behavioral therapy; Psychology.

\section{INTRODUÇÃO}

A droga é qualquer substância química ou mistura de substâncias que tem a capacidade de alterar a função biológica, como humor e cognição, de um organismo vivo ${ }^{1}$. Seu uso tem se tornado mais evidente não só na sociedade brasileira como também no mundo, podendo ser considerado um problema de saúde pública ${ }^{1}$, tendo em vista que o número de usuários dependentes com prejuízos nos âmbitos familiar, social, psicológico, físico e cognitivo teve um crescimento nos últimos anos ${ }^{2,3,4}$. Esses fatos nos levam a estabelecer ainda uma íntima relação da dependência química com o aumento da criminalidade, baixo rendimento escolar e prejuízos nos relacionamento interpessoais e profissionais ${ }^{2}$.

Os motivos que levam as pessoas a fazerem uso são diferentes e podem ser de origem social, psicológica e/ou genética ${ }^{1}$. Dados epidemiológicos ${ }^{5}$ coletados de 107 cidades brasileiras e norte-americanas descreveram que em relação a qualquer droga a prevalência era de $19,4 \%$ no Brasil e 38,9\% nos Estados Unidos. E segundo o Relatório Mundial sobre Drogas, no ano de 2010, aproximadamente 4,6\% da população mundial consumiu algum tipo de substâncias psicoativas (SPA) e dentre esses que fizeram uso, 27 milhões desenvolveram dependências destas substâncias ${ }^{6}$. Já o Relatório Mundial sobre Drogas, no ano de $2016^{7}$ revelou que cerca de 5\% da população adulta, ou 250 milhões de pessoas entre 15 e 64 anos, usou pelo menos uma droga em 2014, mostrando que embora substancial esse número não sofreu elevação, ao longo dos últimos quatro anos, na mesma 
proporção da população mundial, mas indica que o número de pessoas que apresentam transtornos relacionados ao consumo de drogas aumentou desproporcionalmente pela primeira vez em seis anos ${ }^{7}$.

Em relação às modalidades no tratamento, a pesquisa e a prática clínica mostram que a terapia cognitiva se mostra muito eficaz na redução de sintomas e taxas de recorrência, sendo ministrada medicação ou não, em uma ampla variedade de transtornos psiquiátricos ${ }^{8}$. Na aplicação do tratamento de dependência química houve um grande avanço da Terapia cognitivo comportamental (TCC) nas últimas décadas, cujos resultados mostraram-se muito eficazes em vários estudos clínicos ${ }^{9,10}$.

Um estudo ${ }^{11}$ analisou a eficácia da TCC realizando 34 ensaios clínicos em um total de 2340 pacientes e concluiu que dependentes de maconha e de cocaína tem a tendência a se beneficiar mais da TCC, ainda que haja muita relutância por parte dos dependentes químicos em optar pela abstinência e o índice de abandono do tratamento entre os usuários de cocaína seja bastante alto.

Outro estudo ${ }^{12}$ mostra que a TCC vem obtendo avanços significativos no tratamento de dependentes químicos, sobretudo na prevenção de recaídas, contribuindo para que o paciente aprenda a lidar com as situações de risco, tendo em vista a forma com que atua na educação cognitiva auxiliando na flexibilização de respostas.

Essas condições fazem com que as terapias comportamentais e cognitivas ocupem um lugar muito importante na atenção voltada a pessoas que são usuárias de drogas, tendo em vista as técnicas e os resultados comprovados cientificamente, destacando-se assim como uma ferramenta de destaque a esse grupo ${ }^{13}$ por possuir fundamentação teórica e técnicas consistentes ${ }^{14}$.

\section{MATERIAL E MÉTODOS}

Trata-se de uma pesquisa de revisão narrativa, de caráter descritivo, qualitativo, desenvolvido a partir de artigos indexados na Scielo (Scientific Eletronic Libraly Online); Bireme e BVS psi - Biblioteca Virtual em Saúde - Psicologia Brasil, a fim de se identificar publicações recentes envolvendo a temática sobre a eficácia da 
terapia cognitivo comportamental no tratamento da dependência química, perpassando pelas técnicas utilizadas e a eficácia do tratamento.

Para a localização dos artigos publicados na base de dados utilizaram-se os seguintes critérios de inclusão: artigos que relatam o assunto publicado no banco de dados da Scielo (Scientific Electronic Library Online); Bireme; BVS psic e google acadêmico; descritores - palavras-chaves: dependência química e terapia cognitivo comportamental; língua portuguesa; artigos publicados no período de 2007 a 2017.

Os critérios de exclusão foram relacionados aos artigos não disponibilizados na íntegra; artigos repetidos; resenhas; editoriais e dossiês; teses e dissertações; além daqueles que não se enquadravam no ano pesquisado.

A sistematização da coleta de dados iniciou com uma avaliação prévia do material bibliográfico coletado por meio dos resumos, visando identificar os textos que se enquadravam com o tema proposto ou que possuíam assuntos que tinham temas relacionados com a aplicabilidade da terapia cognitivo comportamental em diversas psicopatologias. Além disso, foram selecionados e analisados alguns livros sobre o tema, com o objetivo de elencar e descrever as principais técnicas utilizadas descritas pelos autores mais conceituados na área. Após a seleção realizou-se a leitura dos artigos e capítulos de livro que atendiam aos objetivos do estudo.

\section{RESULTADOS E DISCUSSÃO}

\section{Critérios para abuso de substância}

A dependência de substâncias é identificada sobre os transtornos psiquiátricos como sendo uma doença crônica, que acompanha o indivíduo durante toda a sua vida, sendo que possui tratamento e pode ser controlada ${ }^{15-17}$. Envolve um desejo compulsivo no qual o indivíduo deseja obter a substância a qualquer custo, caracterizando uma necessidade tanto psicológica quanto biológica, englobando um conjunto de sintomas cognitivos, comportamentais e fisiológicos que indicam que o individuo utiliza e/ou segue utilizando determinada substância apesar dos problemas que esta possa causar ${ }^{18}$.

Os critérios para dependência de substância, segundo o DSM V (Manual Diagnóstico Estatístico dos Transtornos Mentais) ${ }^{18}$, baseiam-se em um padrão 
patológico de comportamentos relacionados a seu uso encaixam-se nos agrupamentos gerais de baixo controle, deterioração social, uso arriscado e critérios farmacológicos. Os critérios ${ }^{18}$ para dependência química incluem:

Critério 1 - Consumo de substância em quantidades maiores ou ao longo de um período maior de tempo do que pretendido originalmente.

Critério 2 - Desejo persistente de reduzir ou regular o uso da substância e possível relato de vários esforços mal sucedidos para diminuir ou descontinuar o uso.

Critério 3 - Muito tempo gasto para obter a substância, usá-la ou recuperar-se de seus efeitos, sendo que em alguns casos de transtornos mais graves por uso de substância, praticamente todas as atividades diárias do indivíduo giram em tomo da substância.

Critério 4 - Fissura manifestada por meio de um desejo ou necessidade intensa de usar a droga que podem ocorrer a qualquer momento, mas com maior probabilidade quando em um ambiente onde a droga foi obtida ou usada anteriormente.

Critério 5 - Uso recorrente de substâncias que pode resultar no fracasso em cumprir as principais obrigações no trabalho, na escola ou no lar.

Critério 6 - Uso contínuo da substância apesar de apresentar problemas sociais ou interpessoais persistentes ou recorrentes causados ou exacerbados por seus efeitos.

Critério 7 - Atividades importantes de natureza social, profissional ou recreativa, abandonadas ou reduzidas devido ao uso da substância.

Critério 8 - Uso recorrente da substância em situações que envolvem risco à integridade física.

Critério 9 - Uso contínuo apesar de estar ciente de apresentar um problema físico ou psicológico persistente ou recorrente que provavelmente foi causado ou exacerbado pela substância.

Critério 10 - Tolerância, sinalizada quando uma dose acentuadamente maior da substância é necessária para obter o efeito desejado ou quando um efeito acentuadamente reduzido é obtido após o consumo da dose habitual.

Critério 11 - Abstinência, que é uma síndrome que ocorre quando as concentrações de uma substância no sangue ou nos tecidos diminuem em um 
indivíduo que manteve uso intenso prolongado. Após desenvolver sintomas de abstinência, o indivíduo tende a consumir a substância para aliviá-los ${ }^{18}$.

\section{Terapia cognitiva comportamental}

A terapia cognitiva foi desenvolvida na década de 60 por Aaron Beck e vem apresentando muita eficácia no tratamento de diversos transtornos psiquiátricos, inclusive na dependência química ${ }^{19}$. É uma abordagem diretiva cujo tratamento é realizado num prazo determinado e que tem como pressuposto básico a mudança de pensamentos disfuncionais, que pode ser ministrada de forma estruturada ou semiestruturada ${ }^{20}$. A partir daí, busca-se novas formas de mudança cognitiva nos pacientes, fazendo com que passem a mudar de maneira emocional e comportamental, modificando suas crenças e pensamentos disfuncionais, para que a partir daí haja uma mudança de atitude e comportamento ${ }^{20}$. A figura 1 mostra como se dá o modelo cognitivo-comportamental básico ${ }^{21}$ :
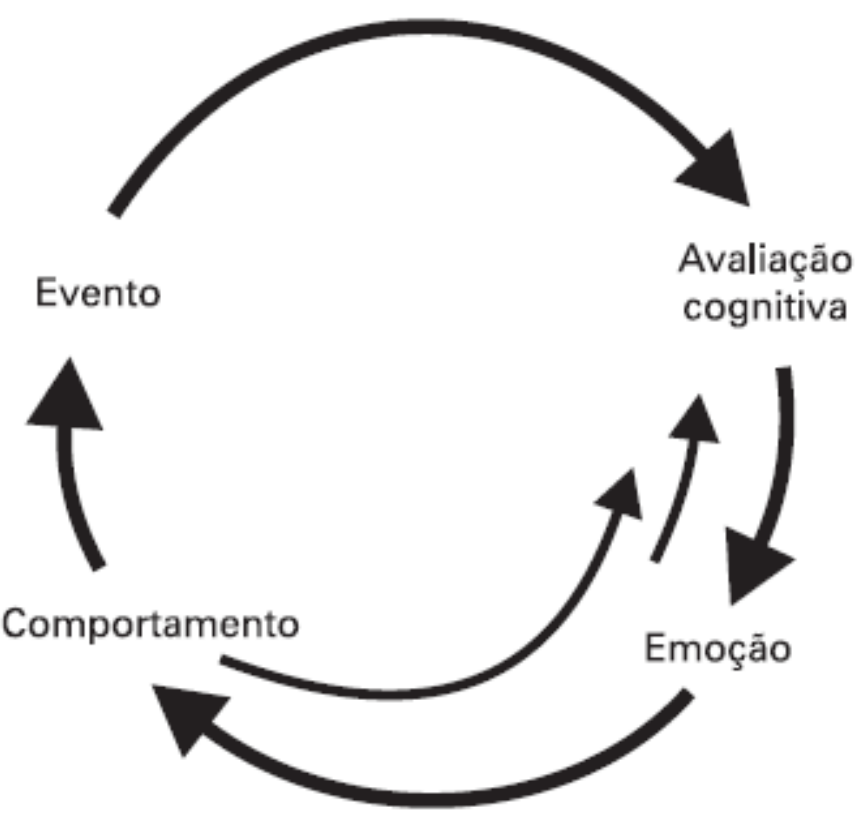

Figura 1 - Modelo cognitivo-comportamental básico ${ }^{21}$

O terapeuta, nesse sentido, procura formular ideias e crenças disfuncionais que o paciente tem sobre si, hipotetizando sobre suas experiências e futuro, para, a partir, daí testar a validade dessas hipóteses por meio de uma 
investigação objetiva e sistemática ${ }^{2}$. Isso oportuniza que o paciente aprenda a avaliar seus pensamentos de forma mais realista e adaptativa e consiga obter uma melhora no seu estado emocional e comportamento ${ }^{22}$ resultando num comportamento mais funcional e fazendo com que o paciente sinta-se melhor ao encarar a situação sob uma nova perspectiva ${ }^{20}$.

A TCC pode ser realizada com pessoas de diferentes níveis de educação e renda, também com pessoas de idades e culturas diferentes ${ }^{23}$. Além disso, nos dias de hoje pode ser usada numa ampla variedade de contextos como escolas, prisões, consultórios, serviços de saúde entre outros, seja em grupo, individual, casal ou família ${ }^{23}$, tendo as seguintes características básicas ${ }^{24}$ :

1. Estilo terapêutico: pressupões que o terapeuta possua várias habilidades sobre a intervenção específica, sendo mais do que alguém que escuta de forma ativa. O terapeuta é ativo e trabalha com o paciente com o objetivo de encontrar informações importantes que servirão de auxílio na resolução dos problemas ${ }^{24}$.

2. Formação psicológica do problema: Não se trata de um diagnóstico, mas sim de uma compreensão de como as experiências são vivenciadas pelo paciente e o que significam para ele $^{24}$.

3. Relação colaborativa: paciente e terapeuta trabalham de forma colaborativa no desenvolvimento de metas, que podem ser revistas sempre que for necessário, sendo que quanto mais específicas, mais úteis serão ${ }^{24}$.

4. Sessões estruturadas: têm a função de desenvolver e manter um componente colaborativo no tratamento. As sessões auxiliam no uso adequado do tempo, onde paciente e terapeuta dividem a responsabilidade pelo seu uso, dando ênfase ao controle e planejamento, o que tende a reduzir distorções cognitivas e comportamentos automáticos que levam a emoções negativas ${ }^{24}$.

5. Orientação de metas: o desenvolvimento de metas é trabalhado de forma colaborativa tanto pelo paciente quanto pelo terapeuta, e as metas podem ser revistas sempre que necessário ${ }^{24}$. 
6. Exame e questionamento de pensamentos: ponto central da TCC, no qual utilizando-se de técnicas e recursos, o terapeuta auxilia o paciente a descobrir respostas mais adaptativas ${ }^{24}$.

7. Disponibilidade de técnicas: a formulação do problema é quem dirige o curso da terapia, e esta vai sendo melhor desenvolvida e compreendida ao longo do tempo, de modo que quanto maior o número de técnicas disponíveis, melhores os resultados ${ }^{24}$.

8. O paciente aprende a ser seu próprio terapeuta: No final do processo o paciente deve estar apto para caminhar sozinho, sendo que o tempo que isso leva, ou quão eficaz a pessoa se sentirá, dependerá de cada paciente. Por isso a importância das tarefas entre as sessões para que o paciente resolva alguns problemas sozinhos ${ }^{24}$.

9. Tarefas para casa: É importante que o paciente esteja disposto e apto para trabalhar entre as sessões, principalmente se o tempo da sessão for semanal ou quinzenal. A tarefa de casa faz com que a mudança se mantenha ativa, fazendo que se sinta responsável por esse processo ${ }^{24}$.

10. Tempo limitado: A terapia tem como meta fazer com que o paciente se sinta autoeficaz para enfrentar situações de risco e não se torne dependente do terapeuta. Esta meta deve ser lembrada a todo momento ao paciente ao longo do tratamento ${ }^{24}$.

A psicoeducação também é uma técnica muita usada na TCC que consiste em informar ao paciente os dados sobre seu diagnóstico, tratamento, funcionamento e prognóstico da doença que o acomete ${ }^{25}$, assim, em vez de contar com o terapeuta para resolver seus problemas, o paciente pode chegar o ponto de identificar as distorções cognitivas e pensamentos desadaptativos para a partir daí reverter esses padrões ${ }^{21}$. A psicoeducação, nesse sentido, provoca o aumento da motivação e estimula a participação pró-ativa do paciente na recuperação ${ }^{26}$.

\section{Modelo cognitivo para dependência de substâncias}

O modelo cognitivo do uso de substâncias baseia-se na situação estímulo, crenças centrais e sobre o uso de drogas, pensamento automático, fissura (craving), crenças permissivas, plano de ação e uso continuado de recaída ${ }^{26}$. As 
técnicas básicas da terapia cognitiva para o abuso de álcool/drogas demandam, em primeiro lugar, fortalecimento da aliança terapêutica através de um entendimento empático do problema do cliente, em combinação com aceitação incondicional ${ }^{27}$.

Situações que estimulam o risco interno e externo desencadeiam crenças centrais sobre o sujeito, o futuro e o mundo no que se refere o uso de drogas, sendo que na maioria das vezes, essas crenças ativadas aparecem de forma inconsciente, dando origem aos pensamentos automáticos ${ }^{22}$. Esse processo leva o sujeito a ir até a droga, iniciar seu uso e a partir daí continuar, mesmo possuindo sentimentos de fracasso e culpa, gerando um desconforto psíquico que por sua vez ativa crenças disfuncionais levando o sujeito a continuar fazendo o uso de substâncias ${ }^{22}$.

O programa SMART (tratamento de recuperação por auto manejo) foi a primeira tentativa de intervenção tendo como base o modelo cognitivo de Albert Elis, e ajuda os indivíduos a atingir a independência dos comportamentos adictivos ${ }^{27}$. O programa fornece instrumentose técnicas para quatro questões programáticas: 1) melhorar e manter a motivação para se abster; 2) enfrentamento de fissuras; 3) resolução de problemas, manejo de pensamentos, sentimentos e comportamentos; e 4) equilíbrio no estilo de vida, balanceando satisfações momentâneas e duradouras ${ }^{27}$.

A partir daí são realizadas intervenções, entrevista motivacional breve, treinamento de habilidades sociais, treinamento de relaxamento, treinamento de autocontrole e da assertividade, manejo do estresse, depressão e ansiedade, reestruturação cognitiva e identificação de situações de alto risco ${ }^{27}$.

\section{Tratamento}

Antes de aplicar o modelo cognitivo para dependência de substâncias objetivando a aplicação de uma série de intervenções, o terapeuta precisa entender até que ponto o indivíduo está pronto para reconhecer ou lidar com um problema de abuso de substância, e para isso são apropriados os métodos tirados da abordagem de entrevista motivacional ${ }^{21}$. Além disso, o sucesso no tratamento demanda o fortalecimento da aliança terapêutica por meio de um entendimento empático do problema do paciente concomitantemente com sua aceitação incondicional, sendo essencial nesse caso explorar o significado e função das ações oposicionistas e 
autodestrutivas do paciente, investigando e avaliando suas crenças sobre a terapia ${ }^{27}$.

O tratamento é direcionado com base nas crenças centrais (CC) intermediárias disfuncionais (Cl) por apresentarem características de baixa tolerância à frustração e estarem relacionadas com a ocorrência de transtornos adictivos $^{19}$. As CC possuem cognições rígidas, gerais e transituacionais, caracterizados por crenças de desamparo e amor como "sou um fracasso", "não sou capaz de ser amado"; já as $\mathrm{Cl}$ podem ser "tudo tem que dar certo pra mim", "as pessoas dificultam as coisas pra mim"19.

As seguintes técnicas podem ser utilizadas no tratamento ${ }^{27}$ :

1. Identificação de pensamentos automáticos (PA): é feito uma lista dos pensamentos distorcidos, visando à reestruturação e identificação de erros cognitivos.

2. Registro diário de pensamentos automáticos disfuncionais: o paciente é orientado a identificar seus pensamentos disfuncionais no final do dia, visando uma análise mais realista de seus pensamentos e sentimento que podem levar o uso da droga.

3. Solução de problemas: ajuda o paciente a adquirir flexibilidade cognitiva ao encontrar soluções para os problemas encontrando soluções (prós e contras).

4. Exame das vantagens e desvantagens: esta técnica permite que o paciente tome alguma decisão por meio da análise das vantagens e desvantagens do uso de drogas, sendo estimulado também a escrever as vantagens e desvantagens da abstinência.

5. Distração: esta técnica permite que o paciente mude o foco de seu mundo interno para o externo ao envolver-se em atividades que lhe deem prazer como, cantar, fazer atividades lúdicas, etc.

6. Cartões de enfrentamento: permitem ao paciente lembrar do que foi discutido na sessão e auxilia na compreensão do processo terapêutico, podendo estar relacionado a vantagens e desvantagens e outras técnicas ${ }^{27}$. 


\section{Prevenção de Recaída}

As abordagens cognitivo-comportamentais ao abuso de substância reconhece que a maioria das pessoas que abusou de substâncias químicas pode sofrer um lapso ou recaída em algum momento da vida ${ }^{21}$. Por isso a Prevenção de Recaída, dentro da TCC, é considerada uma alternativa promissora e eficiente na redução do uso de drogas e é uma técnica que atua na colaboração da mudança de comportamento ${ }^{19}$.

A prevenção de recaída faz um contraponto aos modelos de doença e moral, no qual se admite que a dependência química é um mau hábito adquirido e passível de mudança desde que se explore com afinco as crenças e os comportamentos que fazem com que o indivíduo mantenha seu hábito ${ }^{28}$.

Nesse tipo de tratamento o terapeuta é quem direciona o sujeito ao autogerenciamento, fundamentando-se em modalidades conhecidas como a) conscientização do problema, onde o sujeito reconhece que a ação aditiva causa dificuldades para a sua vida b) o treinamento de habilidades na qual trabalha-se com táticas de como lidar com circunstâncias de risco diante da substância, por meio da análise das competências do sujeito, das que precisam ser aprimoradas e as que precisam ser trabalhadas e c) modificações no estilo de vida: o dependente é convocado a refletir sobre sua atual condição e idealizar um momento ideal sem o consumo das substâncias ${ }^{29}$.

A partir daí pode-se dizer que no modelo de prevenção de recaída há duas possibilidades 1) resposta de enfrentamento: onde o paciente tem um comportamento adequado que o proteja do uso da droga, aumentando assim a avaliação positiva sobre sua capacidade de atingir uma meta e consequentemente diminua a probabilidade de recaída (autoeficácia) ${ }^{30}$ e 2) nenhuma resposta de enfrentamento: no qual as cognições disfuncionais sobre os efeitos das drogas aumentam a probabilidade de recaída, ocasionando assim a diminuição da autoeficácia ${ }^{28}$. 


\section{CONSIDERAÇÕES FINAIS}

Levando em consideração o aumento do número de usuários de substâncias químicas a cada ano e todas as mazelas que advém daí, é imprescindível a adoção de estratégias para enfrentar esse problema que é considerado hoje um problema de saúde pública no mundo inteiro. A Terapia cognitivo-comportamental surge hoje como uma ferramenta poderosa no tratamento de dependentes químicos, tendo sua eficácia comprovada em vários estudos envolvendo a aplicação da TCC nesses casos.

Essa eficácia pode ser comprovada pela fundamentação teórica consistente e pelas técnicas utilizadas, dentre as quais podemos citar a identificação de pensamentos automáticos e a psicoeducação, que contribui para que o paciente seja seu próprio terapeuta. Além disso, a TCC trabalha com a hipótese de que a pessoa pode sofrer uma recaída ao longo do tratamento e por isso dispõe de uma ferramenta conhecida com Prevenção de Recaídas que se configura como uma alternativa eficaz na redução do uso de drogas.

O número de artigos relacionados à aplicação da TCC no tratamento de dependentes químicos encontrados foi reduzido, quando se esperava um número muito maior de artigos relacionados ao tema. A maioria dos artigos encontrados e os livros explicitaram a eficácia da TCC dando ênfase às técnicas utilizadas em casos de abuso de substâncias, enfatizando as técnicas em linhas gerais primeiramente e depois focando na dependência química, principalmente na prevenção de recaídas e no modelo cognitivo para o abuso de substâncias.

\section{REFERÊNCIAS}

1. Bernardi PA, Parizotto APA. Dependência Química e Religiosidade. Pesquisa em Psicologia, 2016; 39-41. [Acesso em 2016 out. 3]. Disponível em:http://editora.unoesc.edu.br/index.php/pp_ae/article/view/11995.

2. Beck JS. Terapia Cognitiva teoria e prática. Porto Alegre: Artes Médicas, 1997.

3. Chalub M, Telles LEB. Álcool, Drogas e Crime. Rev. Bras. Psiquiatr. São Paulo, 2006; 28(2):69-73. 
4. Fontanella BJB. Sintomas psicóticos e cognitivos associados à busca de tratamento por dependentes de substâncias - Um estudo qualitativo. J. bras. psiquiatr. Rio de Janeiro, 2010; 59(2):139-145.

5. Carlini EA, Galduróz JCF, Noto AR, Nappo AS. I Levantamento Domiciliar sobre o Uso de Drogas Psicotrópicas no Brasil: Estudo Envolvendo as 107 Maiores Cidades do País. São Paulo: CEBRID/ Unifesp, 2002.

6. UNODC. Relatório mundial sobre drogas de 2010. United Nations Office ondrugsand crime. [Acesso em 2017 mar 11].Disponível em: http://www.unodc.org/lpo-brazil/pt/frontpage/2010/06/23-relatorio-mundialsobre-drogas-2010-do-unodc-revela-tendencias-de-novas-drogas-e-de-novosmercados.html.

7. UNODC. Relatório mundial sobre drogas de 2016. United Nations Office ondrugsand crime. [Acesso em 2017 mar 11].Disponível em: http://www.unodc.org//po-brazil/pt/drogas/relatorio-mundial-sobre-drogas.html.

8. Knapp P, Beck AT. Fundamentos, modelos conceituais, aplicações e pesquisa em terapia cognitiva. Rev. Bras. Psiquiatr. 2008; 30(Suppl. 2):S54-S64.

9. Silva CJ, Serra AM. Terapias Cognitiva e Cognitivo-Comportamental em dependência química. Rev. Bras. Psiquiatr. São Paulo 2004; 26 (Supl I): 33-39.

10. Knapp P, Luz Junior E, Baldisserotto GV. Terapia cognitiva no tratamento da dependência química. In: Rangé, B. (Org.). Psicoterapias cognitivo comportamentais: um diálogo com a psiquiatria. Porto Alegre: Artmed, 2008. p. 332-350.

11. Dutra I, Stathopoulou G, Basden SI, Leyro TM, Powers MB, Otto MW. A Metaanallytic reviw of psychosocial interventions for substance use disorders. Am J Psychiatry. 2008; 165(2):179-87.

12. Moraes AS. A importância da terapia cognitivo-comportamental no tratamento psicológico do usuário de maconha, uma revisão da literatura. [Monografia]. [São Paulo]: USP; 2013. 44 p. [Acesso em 2017 mar 25]. Disponível em: http://www.uniad.org.br/educacao/apoio-ao-aluno/tccs/item/18156.

13. Morandi M, Guimarães LP. Intervenções Cognitivo-Comportamentais no Tratamento das Dependências Químicas. Id online Rev. Psic. 2015 fev; 9(25):203-2016.

14. Cooper L. Combined motivational interviewing and cognitive-behavioral therapy with older adult drug and alcohol abusers. Health \& Social Work 2012; 37(3):173-179.

15. Pratta EMM, Santos MA. O processo saúde-doença e a dependência química: interfaces e evolução. Psic.: Teor. e Pesq.. Brasília 2009 jun; 25(2):203-211. 
16. Ribeiro $M$, Laranjeira R. $O$ Tratamento do usuário de crack. Porto Alegre: Artmed, 2012. $664 \mathrm{p}$.

17. Zaleski Marcos, Laranjeira Ronaldo Ramos, Marques Ana Cecília Petta Roselli, Ratto Lílian, Romano Marcos, Alves Hamer Nastasy Palhares et al . Diretrizes da Associação Brasileira de Estudos do Álcool e outras Drogas (ABEAD) para o diagnóstico e tratamento de comorbidades psiquiátricas e dependência de álcool e outras substâncias. Rev. Bras. Psiquiatr. 2006 Jun [acesso 2017 Mar 25]; 28(2): 142-148. DOI http://dx.doi.org/10.1590/S151644462006000200013.

18. DSM V. Manual diagnóstico e estatístico de transtornos mentais. Associação Americana de Psiquiatria.Porto Alegre: Artmed, 2014. 948 p.

19. Silva LFM, Branco MFC, Miccione MM. A Eficácia da Terapia CognitivoComportamental no Tratamento da Dependência Química: uma revisão de literatura. Rev. Estação Científica Juiz de Fora 2015 jan/jun; (13):1-17.

20. Beck J. Terapia Cognitivo-Comportamental: Teoria e Prática. Porto Alegre: Artmed, 2013.

21. Wright JH, Sudak DM, Turkington D, Thase ME. Terapia cognitivocomportamental de alto rendimento para sessões breves: Guia ilustrado. Porto Alegre: Artmed, 2012.

22. Luz AAV, Fontana M, Méa CPD. Abordagem Cognitivo Comportamental para Dependência Química. Impacto Científico e Social na Pesquisa. IMED 2015. [acesso 2017 mar 25]. Disponível em: http://soac.imed.edu.br/index.php/mic/ixmic/paper/viewFile/205/44.

23. Beck JS. Cognitivetherapy: Basicsandbeyond (2nd ed.). New York: Guilford, 2011.

24. Simmons J, Griffiths R. CBT for begginers: a praticalguide for begginers. London: Sage, 2009.

25. Farina M, Terroso LB, Lopes RMF, Argimon IIL. Importância da psicoeducação em grupos de dependentes químicos: relato de experiência. Aletheia[Internet]. 2013 Dez [acesso 2017 Mar 18]; (42): 175-185. Disponível em: http://pepsic.bvsalud.org/scielo.php?script=sci_arttext\&pid=S141303942013000300015\&lng=pt.

26. Knapp P. Terapia Cognitivo Comportamental na Prática Psiquiátrica. Porto Alegre: Artmed, 2004.

27. Rangé B, Marlatt GA. Terapia cognitivo-comportamental de transtornos de abuso de álcool e drogas. Rev. Bras. Psiquiatr. 2008; 30 (Supl II):S88-95. [acesso 2017 mar 25]. Disponível em: http://www.scielo.br/pdf/rbp/v30s2/a06v30s2.pdf. 
28. Marlatt GA, Gordon GR. Prevenção da Recaída. Porto Alegre: Artes Médicas; 1994.

29. Jungerman FS. Prevenção De Recaída. In: Cordeiro DC, Figlie NF, Laranjeira R. Boas Práticas no Tratamento do Uso e Dependência de Substâncias. São Paulo: Roca, 2007.

30. Daley CD, Marlatt AG. RelapsePrevention. In: Lowinson JH, Ruiz P, Millman RB, Langrod JG, editors. Sustance Abuse. A ComprehensiveTextbook. 3rd ed. New York: Williams \& Wilkins; 1997. p. 458-67. 